\title{
Modified Decomposition Method with New Inverse Differential Operators for Solving Singular Nonlinear IVPs in First- and Second-Order PDEs Arising in Fluid Mechanics
}

\author{
Nemat Dalir \\ Department of Mechanical Engineering, Salmas Branch, Islamic Azad University, Salmas, Iran \\ Correspondence should be addressed to Nemat Dalir; dalir@aut.ac.ir
}

Received 15 February 2014; Accepted 6 June 2014; Published 19 June 2014

Academic Editor: Onesimo Hernandez-Lerma

Copyright (C) 2014 Nemat Dalir. This is an open access article distributed under the Creative Commons Attribution License, which permits unrestricted use, distribution, and reproduction in any medium, provided the original work is properly cited.

Singular nonlinear initial-value problems (IVPs) in first-order and second-order partial differential equations (PDEs) arising in fluid mechanics are semianalytically solved. To achieve this, the modified decomposition method (MDM) is used in conjunction with some new inverse differential operators. In other words, new inverse differential operators are developed for the MDM and used with the MDM to solve first- and second-order singular nonlinear PDEs. The results of the solutions by the MDM together with new inverse operators are compared with the existing exact analytical solutions. The comparisons show excellent agreement.

\section{Introduction}

Singular nonlinear partial differential equations (PDEs) arise in various physical phenomena in applied sciences and engineering from such areas as fluid mechanics and heat transfer, Riemannian geometry, applied probability, mathematical physics, and biology. The Adomian decomposition method (ADM) and modified decomposition method $(\mathrm{MDM})$ are semianalytical methods that give approximate analytical solutions for the differential equations. MDM was first developed by Wazwaz and El-Seyed [1] who applied it to solve the ordinary differential equations (ODEs). Since then the MDM has been used for solving various equations in mathematics and physics [2-4], boundary value problems [59], various problems in engineering [10-13], and initial-value problems [14-17]. Adomian et al. [14] solved the Lane-Emden equation using the MDM. Wazwaz [15] investigated singular initial-value problems, linear and nonlinear, homogeneous and nonhomogeneous, by using the ADM. Hasan and Zhu [16] reported the solution of singular nonlinear initial-value problems in ordinary differential equations (ODEs) by the ADM. Wu [17] extended the ADM for the calculations of the nondifferentiable functions in nonsmooth initial-value problems. His iteration procedure was based on Jumarie Taylor series. Abassy [18] introduced a qualitative improvement in the ADM for solving nonlinear nonhomogenous initial-value problems. Lin et al. [19], based on a new definition of the Adomian polynomials and the two-step Adomian decomposition method combined with the Pade technique, proposed a new algorithm to construct accurate analytical approximations of nonlinear differential equations with initial conditions. Wazwaz et al. [20] used the ADM to handle the integral form of the Lane-Emden equations with initial values and boundary conditions.

To the best knowledge of author, till now, no one has attempted the modified decomposition method on solving singular nonlinear partial differential equations. Our motivation in the present study is to improve the MDM by new developed inverse differential operators to obtain approximate analytical solutions to the singular nonlinear initial-value problems in first- and second-order PDEs.

\section{Application of MDM for Solving Singular Nonlinear PDEs}

2.1. General First-Order Singular Nonlinear PDEs. Consider the following general first-order (in $t$ ) singular nonlinear PDE:

$$
\frac{\partial u}{\partial t}+\frac{p}{t} u=F\left(x, u, \frac{\partial u}{\partial x}\right)
$$


where $t$ and $x$ are independent variables, $u$ is the dependent variable, $F$ is a nonlinear function of $x, u$, and $u_{x}$, and $p$ is a real constant: $p>0$. The initial condition is as follows:

$$
u(x, 0)=h(x) .
$$

In order to solve the PDE (1) with initial condition (2) by the modified decomposition method, at first, the linear differential operator $L_{t}(\cdot)=\partial(\cdot) / \partial t+(p / t)(\cdot)$ is defined, and the left-hand side of $(1)$ is rewritten as

$$
L_{t} u=\frac{\partial u}{\partial t}+\frac{p}{t} u
$$

The inverse differential operator of $L_{t}$, that is, $L_{t}^{-1}$, is defined such that $L_{t}^{-1}\left\{L_{t}(u)\right\}=u$ :

$$
L_{t}^{-1}(\cdot)=\frac{1}{t^{p}} \int_{0}^{t} t^{p}(\cdot) d t
$$

It can be shown that applying the inverse differential operator, defined in (4), to the left-hand side of (1) results in

$$
\begin{aligned}
& L_{t}^{-1}\left(\frac{\partial u}{\partial t}+\frac{p}{t} u\right) \\
& \quad=\frac{1}{t^{p}} \int_{0}^{t} t^{p}\left(\frac{\partial u}{\partial t}+\frac{p}{t} u\right) d t=\frac{1}{t^{p}} \int_{0}^{t}\left(t^{p} \frac{\partial u}{\partial t}+p t^{p-1} u\right) d t \\
& \quad=\frac{1}{t^{p}} \int_{0}^{t} \frac{\partial\left(t^{p} u\right)}{\partial t} d t=\frac{1}{t^{p}}\left(t^{p} u\right)_{0}^{t}=\frac{1}{t^{p}} t^{p} u=u .
\end{aligned}
$$

The inverse differential operator of (4), defined in the present work, can be used to solve the general first-order singular nonlinear PDEs. Applying (4) to (1) gives

$$
\begin{aligned}
L_{t} u & =F\left(x, u, \frac{\partial u}{\partial x}\right) \stackrel{L_{t}^{-1}}{\longrightarrow} u(x, t) \\
& =h(x)+L_{t}^{-1}\left(F\left(x, u, \frac{\partial u}{\partial x}\right)\right),
\end{aligned}
$$

where $h(x)$ is obtained as the result of initial condition. The Adomian decomposition method (ADM) states that the dependent variable $u(x, t)$ and the nonlinear term $F$ should be written as the following infinite series [1]:

$$
\begin{aligned}
u(x, t) & =\sum_{m=0}^{\infty} u_{m}(x, t), \\
F\left(x, u, \frac{\partial u}{\partial x}\right) & =\sum_{m=0}^{\infty} A_{m}(x, t) .
\end{aligned}
$$

Substituting the infinite series of (7) in (6) gives

$$
\sum_{m=0}^{\infty} u_{m}(x, t)=h(x)+L_{t}^{-1}\left(\sum_{m=0}^{\infty} A_{m}(x, t)\right)
$$

According to the ADM, all terms of $u(x, t)$ except $u_{0}(x, t)$ are determined by recursive relation; that is, [2]

$$
\begin{aligned}
u_{0}(x, t) & =h(x), \\
u_{1}(x, t) & =L_{t}^{-1}\left(A_{0}(x, t)\right), \\
u_{m+1}(x, t) & =L_{t}^{-1}\left(A_{m}(x, t)\right), \quad m \geq 1 .
\end{aligned}
$$

The modified decomposition method (MDM) applies a slight modification to ADM, such that it splits $h(x)$ into two parts: $h(x)=h_{1}(x)+h_{2}(x)$; the first part, $h_{1}(x)$, is written with $u_{0}(x, t)$, and the second part, $h_{2}(x)$, is written with $u_{1}(x, t)$ as follows:

$$
\begin{aligned}
u_{0}(x, t) & =h_{1}(x), \\
u_{1}(x, t) & =h_{2}(x)+L_{t}^{-1}\left(A_{0}(x, t)\right), \\
u_{m+1}(x, t) & =L_{t}^{-1}\left(A_{m}(x, t)\right), \quad m \geq 1 .
\end{aligned}
$$

Although modification to ADM from MDM is slight, it enhances convergence behavior of the decomposition method. The Adomian polynomials $A_{m}$ 's are defined as [3]

$$
A_{m}=\left[\frac{1}{m !} \frac{d^{m}}{d \lambda^{m}} F\left(\sum_{i=0}^{m} \lambda^{i} u_{i}\right)\right]_{\lambda=0} .
$$

2.2. Second-Order Singular Nonlinear PDEs. Consider the following second-order (in $t$ ) singular nonlinear partial differential equation:

$$
\frac{\partial^{2} u}{\partial t^{2}}+\frac{2}{t} \frac{\partial u}{\partial t}=F\left(x, u, \frac{\partial u}{\partial x}, \frac{\partial^{2} u}{\partial x^{2}}\right)
$$

where $t$ and $x$ are independent variables, $u$ is the dependent variable, and $F$ is a nonlinear function of $x, u, u_{x}$, and $u_{x x}$. The initial conditions for the PDE (12) are as follows:

$$
u(x, 0)=f(x), \quad u_{t}(x, 0)=g(x) .
$$

In order to use the MDM, the left-hand side of (12) is considered as the linear invertible operator $L_{t}$ :

$$
L_{t} u=\frac{\partial^{2} u}{\partial t^{2}}+\frac{2}{t} \frac{\partial u}{\partial t} \Longrightarrow L_{t}(\cdot)=\frac{\partial^{2}(\cdot)}{\partial t^{2}}+\frac{2}{t} \frac{\partial(\cdot)}{\partial t} .
$$

The inverse of the linear differential operator $L_{t}$, that is, $L_{t}^{-1}$, is defined as [16]

$$
L_{t}^{-1}(\cdot)=\frac{1}{t} \int_{0}^{t} \int_{0}^{t} t(\cdot) d t d t
$$


Applying the inverse differential operator $L_{t}^{-1}$, defined in (15), to the left-hand side of (12) gives

$$
\begin{aligned}
& L_{t}^{-1}\left(\frac{\partial^{2} u}{\partial t^{2}}+\frac{2}{t} \frac{\partial u}{\partial t}\right) \\
& \quad=\frac{1}{t} \int_{0}^{t} \int_{0}^{t} t\left(\frac{\partial^{2} u}{\partial t^{2}}+\frac{2}{t} \frac{\partial u}{\partial t}\right) d t d t \\
& =\frac{1}{t} \int_{0}^{t} \int_{0}^{t}\left(t \frac{\partial^{2} u}{\partial t^{2}}+2 \frac{\partial u}{\partial t}\right) d t d t \\
& =\frac{1}{t} \int_{0}^{t} \int_{0}^{t} \frac{\partial}{\partial t}\left(t \frac{\partial u}{\partial t}+u\right) d t d t \\
& =\frac{1}{t} \int_{0}^{t}\left(t \frac{\partial u}{\partial t}+u-u(x, 0)\right) d t \\
& =\frac{1}{t} \int_{0}^{t} \frac{\partial}{\partial t}(t u) d t-u(x, 0) \\
& =\frac{1}{t}(t u)_{0}^{t}-u(x, 0)=u(x, t)-u(x, 0) .
\end{aligned}
$$

The inverse differential operator of (15) can be used to solve the second-order singular nonlinear PDEs. Applying the inverse differential operator of (15) to (12) results in

$$
\begin{aligned}
L_{t} u & =F\left(x, u, \frac{\partial u}{\partial x}, \frac{\partial^{2} u}{\partial x^{2}}\right) \stackrel{L_{t}^{-1}}{\longrightarrow} u(x, t) \\
& =f(x)+\operatorname{tg}(x)+L_{t}^{-1}\left(F\left(x, u, \frac{\partial u}{\partial x}, \frac{\partial^{2} u}{\partial x^{2}}\right)\right),
\end{aligned}
$$

where $f(x)+\operatorname{tg}(x)$ is obtained as the result of initial conditions. Substitution of the dependent variable $u(x, t)$ and the nonlinear term $F$ with the infinite series of (7) gives

$$
\sum_{m=0}^{\infty} u_{m}(x, t)=f(x)+\operatorname{tg}(x)+L_{t}^{-1}\left(\sum_{m=0}^{\infty} A_{m}(x, t)\right),
$$

where the Adomian polynomials, $A_{m}$ 's, are defined in (11). The modified decomposition method splits $f(x)+\operatorname{tg}(x)$ into two parts; the first part, $f(x)$, is written with $u_{0}(x, t)$, and the second part, $\operatorname{tg}(x)$, is written with $u_{1}(x, t)$ as follows:

$$
\begin{aligned}
u_{0}(x, t) & =f(x), \\
u_{1}(x, t) & =\operatorname{tg}(x)+L_{t}^{-1}\left(A_{0}(x, t)\right), \\
u_{m+1}(x, t) & =L_{t}^{-1}\left(A_{m}(x, t)\right), \quad m \geq 1 .
\end{aligned}
$$

2.3. General Second-Order Singular Nonlinear PDEs. Consider the following general second-order (in $t$ ) singular nonlinear PDE:

$$
\frac{\partial^{2} u}{\partial t^{2}}+\frac{p}{t} \frac{\partial u}{\partial t}=F\left(x, u, \frac{\partial u}{\partial x}, \frac{\partial^{2} u}{\partial x^{2}}\right)
$$

where $t$ and $x$ are independent variables, $u$ is the dependent variable, $F$ is a nonlinear function of $x, u, u_{x}$, and $u_{x x}$, and $p$ is a real constant: $p>0$. The initial conditions for the PDE (20) are

$$
u(x, 0)=f(x), \quad u_{t}(x, 0)=g(x) .
$$

In order to use the MDM, the left-hand side of PDE (20) is considered as the linear invertible operator $L_{t}$ :

$$
L_{t} u=\frac{\partial^{2} u}{\partial t^{2}}+\frac{p}{t} \frac{\partial u}{\partial t} \Longrightarrow L_{t}(\cdot)=\frac{\partial^{2}(\cdot)}{\partial t^{2}}+\frac{p}{t} \frac{\partial(\cdot)}{\partial t} .
$$

The inverse of the linear differential operator $L_{t}$, that is, $L_{t}^{-1}$, is defined as

$$
L_{t}^{-1}(\cdot)=\int_{0}^{t} \frac{1}{t^{p}} \int_{0}^{t} t^{p}(\cdot) d t d t
$$

It can be shown in the following manner that if $L_{t}^{-1}$ of (23) is applied to the left-hand side of $(20)$, it gives $u(x, t)-u(x, 0)$ :

$$
\begin{aligned}
& L_{t}^{-1}\left(\frac{\partial^{2} u}{\partial t^{2}}+\frac{p}{t} \frac{\partial u}{\partial t}\right) \\
& \quad=\int_{0}^{t} \frac{1}{t^{p}} \int_{0}^{t} t^{p}\left(\frac{\partial^{2} u}{\partial t^{2}}+\frac{p}{t} \frac{\partial u}{\partial t}\right) d t d t \\
& \quad=\int_{0}^{t} \frac{1}{t^{p}} \int_{0}^{t}\left(t^{p} \frac{\partial^{2} u}{\partial t^{2}}+p t^{p-1} \frac{\partial u}{\partial t}\right) d t d t \\
& \quad=\int_{0}^{t} \frac{1}{t^{p}} \int_{0}^{t} \frac{\partial}{\partial t}\left(t^{p} \frac{\partial u}{\partial t}\right) d t d t \\
& \quad=\int_{0}^{t} \frac{1}{t^{p}}\left(t^{p} \frac{\partial u}{\partial t}\right) d t \\
& \quad=\int_{0}^{t} \frac{\partial u}{\partial t} d t=(u)_{0}^{t}=u(x, t)-u(x, 0) .
\end{aligned}
$$

The inverse differential operator of (23), obtained here for the general second-order singular nonlinear PDEs, has never been reported before. Applying the inverse differential operator of (23) to (31) results in

$$
\begin{aligned}
L_{t} u & =F\left(x, u, \frac{\partial u}{\partial x}, \frac{\partial^{2} u}{\partial x^{2}}\right) \stackrel{L_{t}^{-1}}{\longrightarrow} u(x, t) \\
& =f(x)+\operatorname{tg}(x)+L_{t}^{-1}\left(F\left(x, u, \frac{\partial u}{\partial x}, \frac{\partial^{2} u}{\partial x^{2}}\right)\right),
\end{aligned}
$$

where $f(x)+\operatorname{tg}(x)$ is obtained as the result of initial conditions. Substitution of the dependent variable $u(x, t)$ and the nonlinear term $F$ with the infinite series of (7) in (25) gives

$$
\sum_{m=0}^{\infty} u_{m}(x, t)=f(x)+\operatorname{tg}(x)+L_{t}^{-1}\left(\sum_{m=0}^{\infty} A_{m}(x, t)\right),
$$

where the Adomian polynomials $A_{m}$ 's are defined in (11). The MDM splits $f(x)+\operatorname{tg}(x)$ into two parts; the first part, $f(x)$, 
is written with $u_{0}(x, t)$, and the second part, $\operatorname{tg}(x)$, is written with $u_{1}(x, t)$ as follows:

$$
\begin{aligned}
u_{0}(x, t) & =f(x), \\
u_{1}(x, t) & =\operatorname{tg}(x)+L_{t}^{-1}\left(A_{0}(x, t)\right), \\
u_{m+1}(x, t) & =L_{t}^{-1}\left(A_{m}(x, t)\right), \quad m \geq 1 .
\end{aligned}
$$

2.4. General Complete Second-Order Singular Nonlinear PDEs. Consider the general second-order (in $t$ ) singular nonlinear PDE in following form:

$$
\frac{\partial^{2} u}{\partial t^{2}}+\frac{2 p}{t} \frac{\partial u}{\partial t}+\frac{p(p-1)}{t^{2}} u=F\left(x, u, \frac{\partial u}{\partial x}, \frac{\partial^{2} u}{\partial x^{2}}, \frac{\partial u}{\partial t}\right)
$$

where $t$ and $x$ are independent variables, $u$ is the dependent variable, $F$ is a nonlinear function of $x, u, u_{x}, u_{x x}$, and $u_{t}$, and $p$ is a real constant: $p>1$. The initial conditions are as follows:

$$
u(x, 0)=f(x), \quad u_{t}(x, 0)=g(x) .
$$

Defining the linear differential operator $L_{t}(\cdot)=\left(\partial^{2}(\cdot) / \partial t^{2}\right)+$ $((2 p / t)(\partial(\cdot) / \partial t))+\left(\left(p(p-1) / t^{2}\right)(\cdot)\right)$, the left-hand side of $(29)$ is rewritten as

$$
L_{t} u=\frac{\partial^{2} u}{\partial t^{2}}+\frac{2 p}{t} \frac{\partial u}{\partial t}+\frac{p(p-1)}{t^{2}} u .
$$

The inverse differential operator of $L_{t}$, that is, $L_{t}^{-1}$, is defined such that $L_{t}^{-1}\left\{L_{t}(u)\right\}=u[16]$ :

$$
L_{t}^{-1}(\cdot)=\frac{1}{t^{p}} \int_{0}^{t} \int_{0}^{t} t^{p}(\cdot) d t d t
$$

Following the definition of inverse differential operator $L_{t}^{-1}$, that is, (31), it can be shown that

$$
\begin{aligned}
& L_{t}^{-1}\left(\frac{\partial^{2} u}{\partial t^{2}}+\frac{2 p}{t} \frac{\partial u}{\partial t}+\frac{p(p-1)}{t^{2}} u\right) \\
& \quad=\frac{1}{t^{p}} \int_{0}^{t} \int_{0}^{t} t^{p}\left(\frac{\partial^{2} u}{\partial t^{2}}+\frac{2 p}{t} \frac{\partial u}{\partial t}+\frac{p(p-1)}{t^{2}} u\right) d t d t \\
& \quad=\frac{1}{t^{p}} \int_{0}^{t} \int_{0}^{t}\left(t^{p} \frac{\partial^{2} u}{\partial t^{2}}+2 p t^{p-1} \frac{\partial u}{\partial t}+p(p-1) t^{p-2} u\right) d t d t \\
& \quad=\frac{1}{t^{p}} \int_{0}^{t} \int_{0}^{t} \frac{\partial}{\partial t}\left(t^{p} \frac{\partial u}{\partial t}+p t^{p-1} u\right) d t d t \\
& \quad=\frac{1}{t^{p}} \int_{0}^{t}\left(t^{p} \frac{\partial u}{\partial t}+p t^{p-1} u\right) d t \\
& \quad=\frac{1}{t^{p}} \int_{0}^{t} \frac{\partial}{\partial t}\left(t^{p} u\right) d t=\frac{1}{t^{p}}\left(t^{p} u\right)=u .
\end{aligned}
$$

The inverse differential operator of (31) can be used to solve the general complete second-order singular nonlinear PDEs.
Applying the inverse differential operator of (31) to (28) results in

$$
\begin{aligned}
L_{t} u & =F\left(x, u, \frac{\partial u}{\partial x}, \frac{\partial^{2} u}{\partial x^{2}}, \frac{\partial u}{\partial t}\right) \stackrel{L_{t}^{-1}}{\longrightarrow} u(x, t) \\
& =f(x)+\operatorname{tg}(x)+L_{t}^{-1}\left(F\left(x, u, \frac{\partial u}{\partial x}, \frac{\partial^{2} u}{\partial x^{2}}, \frac{\partial u}{\partial t}\right)\right),
\end{aligned}
$$

where $f(x)+\operatorname{tg}(x)$ appears as the result of initial conditions. Using the MDM, (33) can be rewritten as [11]

$$
\sum_{m=0}^{\infty} u_{m}(x, t)=f(x)+\operatorname{tg}(x)+L_{t}^{-1}\left(\sum_{m=0}^{\infty} A_{m}(x, t)\right)
$$

where the Adomian polynomials $A_{m}$ 's are defined in (11). The MDM splits $f(x)+\operatorname{tg}(x)$ into two parts; $f(x)$ is written with $u_{0}(x, t)$ and $t g(x)$ is written with $u_{1}(x, t)$ as follows:

$$
\begin{aligned}
u_{0}(x, t) & =f(x), \\
u_{1}(x, t) & =\operatorname{tg}(x)+L_{t}^{-1}\left(A_{0}(x, t)\right), \\
u_{m+1}(x, t) & =L_{t}^{-1}\left(A_{m}(x, t)\right), \quad m \geq 1 .
\end{aligned}
$$

\section{Case Studies}

\subsection{Case Study for First-Order Singular Nonlinear PDEs}

Case Study 1. Consider the following first-order (in $t$ ) nonhomogeneous singular nonlinear PDE with a homogeneous initial condition:

$$
\begin{aligned}
& u_{t}+\frac{u}{2 t}=6 u \cdot u_{x}-u_{x x x}-\frac{7}{2} t^{2}+9 t^{3}, \\
& u(x, 0)=0 .
\end{aligned}
$$

Applying the inverse differential operator $L_{t}^{-1}(\cdot)=\left(1 / t^{1 / 2}\right)$ $\int_{0}^{t} t^{1 / 2}(\cdot) d t$, defined in (4) with $p=1 / 2$, on (36) gives

$$
u(x, t)=-t^{3}+2 t^{4}+6 L_{t}^{-1}\left(u \cdot u_{x}\right)-L_{t}^{-1}\left(u_{x x x}\right) .
$$

Now, according to the MDM, the dependent variable $u(x, t)$ and the nonlinear term $u \cdot u_{x}$ are substituted with the infinite series of (7) in (37) as follows:

$$
\left\{\begin{aligned}
u(x, t)= & \sum_{m=0}^{\infty} u_{m}(x, t), \\
u \cdot u_{x}= & \sum_{m=0}^{\infty} A_{m}(x, t) \\
\Longrightarrow \sum_{m=0}^{\infty} u_{m}(x, t)= & -t^{3}+2 t^{4}+6 L_{t}^{-1} \\
& \quad \times\left(\sum_{m=0}^{\infty} A_{m}(x, t)\right) \\
& -L_{t}^{-1}\left(u_{m_{x x x}}\right) .
\end{aligned}\right.
$$


Due to the MDM in (10), $u_{0}, u_{1}$, and the recursive relation for $u_{m+1}$ are obtained from (38) as

$$
\begin{aligned}
u_{0}(x, t) & =-t^{3}, \\
u_{1}(x, t) & =2 t^{4}+6 L_{t}^{-1}\left(A_{0}(x, t)\right)-L_{t}^{-1}\left(u_{0_{x x x}}\right), \\
u_{m+1}(x, t) & =6 L_{t}^{-1}\left(A_{m}(x, t)\right)+L_{t}^{-1}\left(u_{m_{x x x}}\right), \quad m \geq 1 .
\end{aligned}
$$

The Adomian polynomials $A_{m}$ 's, according to (11), are obtained as

$$
\begin{aligned}
& A_{0}(x, t)=u_{0}(x, t) \cdot u_{0_{x}}(x, t)=0, \\
& A_{1}(x, t)=u_{0}(x, t) \cdot u_{1_{x}}(x, t)+u_{1}(x, t) \cdot u_{0_{x}}(x, t)=0 \text {, } \\
& A_{2}(x, t)=u_{0}(x, t) \cdot u_{2_{x}}(x, t)+u_{1}(x, t) \cdot u_{1_{x}}(x, t) \\
& +u_{2}(x, t) \cdot u_{0_{x}}(x, t)=0, \\
& A_{m}(x, t)=0, \quad m \geq 3 \text {, }
\end{aligned}
$$

and the expressions for $u_{m}$ 's from (39) become

$$
\begin{gathered}
u_{0}(x, t)=-t^{3}, \\
u_{1}(x, t)=2 t^{4}, \\
u_{2}(x, t)=6 L_{t}^{-1}\left(A_{1}(x, t)\right)+L_{t}^{-1}\left(u_{1_{x x x}}\right)=0, \\
u_{m}(x, t)=0, \quad m \geq 3 .
\end{gathered}
$$

The solution of the first-order singular nonlinear initial-value problem of (36) by the use of MDM is the sum of $u_{m}$; that is, $u(x, t)=\sum_{m=0}^{\infty} u_{m}(x, t)$, such that

$$
u(x, t)=u_{0}(x, t)+u_{1}(x, t)+\cdots=-t^{3}+2 t^{4}
$$

which is the exact solution of the initial-value problem of (36) given by the MDM, which shows the precision of the MDM with the new developed inverse differential operator.

\subsection{Case Studies for Second-Order Singular Nonlinear PDEs}

Case Study 2. Consider the following second-order initialvalue problem:

$$
\begin{gathered}
\frac{\partial^{2} u}{\partial t^{2}}+\frac{2}{t} \frac{\partial u}{\partial t}+\frac{\partial u}{\partial x} \cdot \frac{\partial^{2} u}{\partial x^{2}}=1+x \\
u(x, 0)=0, \quad u_{t}(x, 0)=0 .
\end{gathered}
$$

Application of the inverse differential operator $L_{t}^{-1}(\cdot)=$ $(1 / t) \int_{0}^{t} \int_{0}^{t} t(\cdot) d t d t$, defined in (15), on the PDE (43) gives

$$
u(x, t)=\frac{t^{2}}{6}+\frac{t^{2}}{6} x-L_{t}^{-1}\left(u_{x} \cdot u_{x x}\right)
$$

Using the MDM with (44) results in

$$
\begin{aligned}
& \sum_{m=0}^{\infty} u_{m}(x, t) \\
& =\frac{t^{2}}{6}+\frac{t^{2}}{6} x-L_{t}^{-1}\left(\sum_{m=0}^{\infty} A_{m}(x, t)\right) \\
& \Longrightarrow\left\{\begin{array}{l}
u_{0}(x, t)=\frac{t^{2}}{6}, \\
u_{1}(x, t)=\frac{t^{2}}{6} x-L_{t}^{-1}\left(A_{0}(x, t)\right), \\
u_{m+1}(x, t)=-L_{t}^{-1}\left(A_{m}(x, t)\right), \quad m \geq 1 .
\end{array}\right.
\end{aligned}
$$

The expressions for $A_{m}$ 's can be expressed as follows:

$$
\begin{aligned}
& A_{0}(x, t)=u_{0_{x}}(x, t) \cdot u_{0_{x x}}(x, t)=0, \\
& A_{1}(x, t)=u_{0_{x}}(x, t) \cdot u_{1_{x x}}(x, t)+u_{1_{x}}(x, t) \cdot u_{0_{x x}}(x, t)=0 \text {, } \\
& A_{2}(x, t)=u_{0_{x}}(x, t) \cdot u_{2_{x x}}(x, t)+u_{1_{x}}(x, t) \cdot u_{1_{x x}}(x, t) \\
& +u_{2_{x}}(x, t) \cdot u_{0_{x x}}(x, t)=0 \text {, } \\
& A_{m}(x, t)=0, \quad m \geq 3 \text {, }
\end{aligned}
$$

and the expressions for $u_{m}$ 's become

$$
\begin{aligned}
& u_{0}(x, t)=\frac{t^{2}}{6}, \\
& u_{1}(x, t)=\frac{t^{2}}{6} x, \\
& u_{2}(x, t)=-L_{t}^{-1}\left(A_{1}(x, t)\right)=0, \\
& u_{m}(x, t)=-L_{t}^{-1}\left(A_{m-1}(x, t)\right)=0, \quad m \geq 3 .
\end{aligned}
$$

Therefore, solution of second-order initial-value problem of (43) by MDM is as follows:

$$
u(x, t)=u_{0}(x, t)+u_{1}(x, t)+\cdots=(x+1) \frac{t^{2}}{6},
$$

which is the exact solution of the initial-value problem of (43).

Case Study 3. Consider the following general second-order nonhomogeneous initial-value problem with the homogeneous initial conditions:

$$
\begin{gathered}
\frac{\partial^{2} u}{\partial t^{2}}-\frac{1}{3 t} \frac{\partial u}{\partial t}-\left(\frac{\partial u}{\partial x}\right)^{2}=1+\frac{5}{3} x t-t^{2} \\
u(x, 0)=0, \quad u_{t}(x, 0)=0 .
\end{gathered}
$$

Application of the inverse differential operator $L_{t}^{-1}(\cdot)=$ $\int_{0}^{t} t^{1 / 3} \int_{0}^{t} t^{-1 / 3}(\cdot) d t d t$, defined in (23) with $p=-1 / 3$, on PDE (49) gives

$$
u(x, t)=\frac{3}{4} t^{2}+\frac{x}{3} t^{3}-\frac{3}{32} t^{4}+L_{t}^{-1}\left(\left(u_{x}\right)^{2}\right) .
$$


Use of MDM results in

$$
\begin{aligned}
& \sum_{m=0}^{\infty} u_{m}(x, t) \\
& =\frac{3}{4} t^{2}+\frac{x}{3} t^{3}-\frac{3}{32} t^{4}+L_{t}^{-1}\left(\sum_{m=0}^{\infty} A_{m}(x, t)\right) \\
& \Longrightarrow\left\{\begin{array}{l}
u_{0}(x, t)=\frac{3}{4} t^{2}, \\
u_{1}(x, t)=\frac{x}{3} t^{3}-\frac{3}{32} t^{4}+L_{t}^{-1}\left(A_{0}(x, t)\right), \\
u_{m+1}(x, t)=L_{t}^{-1}\left(A_{m}(x, t)\right), \quad m \geq 1 .
\end{array}\right.
\end{aligned}
$$

The expressions for $A_{m}$ 's are as follows:

$$
\begin{aligned}
A_{0}(x, t)= & \left(u_{0_{x}}(x, t)\right)^{2}=0, \\
A_{1}(x, t)= & 2 u_{0_{x}}(x, t) \cdot u_{1_{x}}(x, t)=0, \\
A_{2}(x, t)= & 2 u_{0_{x}}(x, t) \cdot u_{2_{x}}(x, t)+\left(u_{1_{x}}(x, t)\right)^{2}=\frac{t^{6}}{9}, \\
A_{3}(x, t)= & 2 u_{0_{x}}(x, t) \cdot u_{3_{x}}(x, t)+2 u_{1_{x}}(x, t) \cdot u_{2_{x}}(x, t)=0, \\
A_{4}(x, t)= & 2 u_{0_{x}}(x, t) \cdot u_{4_{x}}(x, t) \\
& +2 u_{1_{x}}(x, t) \cdot u_{3_{x}}(x, t)\left(u_{2_{x}}(x, t)\right)^{2}=0, \\
A_{m}(x, t)= & 0, \quad m \geq 5,
\end{aligned}
$$

and the expressions for $u_{m}$ 's take the following forms:

$$
\begin{aligned}
& u_{0}(x, t)=\frac{3}{4} t^{2} \\
& u_{1}(x, t)=\frac{x}{3} t^{3}-\frac{3}{32} t^{4}, \\
& u_{2}(x, t)=L_{t}^{-1}\left(A_{1}(x, t)\right)=0, \\
& u_{3}(x, t)=L_{t}^{-1}\left(A_{2}(x, t)\right)=\frac{1}{460} t^{23 / 3}, \\
& u_{4}(x, t)=L_{t}^{-1}\left(A_{3}(x, t)\right)=0, \\
& u_{5}(x, t)=L_{t}^{-1}\left(A_{4}(x, t)\right)=0, \\
& u_{m}(x, t)=L_{t}^{-1}\left(A_{m-1}(x, t)\right)=0, \quad m \geq 6 .
\end{aligned}
$$

Thus the solution of the second-order initial-value problem of (49) by MDM is as follows:

$$
\begin{aligned}
u(x, t) & =u_{0}(x, t)+u_{1}(x, t)+u_{2}(x, t)+u_{3}(x, t)+\cdots \\
& =\frac{3}{4} t^{2}+\frac{x}{3} t^{3}-\frac{3}{32} t^{4}+\frac{1}{460} t^{23 / 3}
\end{aligned}
$$

It is again seen that the MDM gives exact solution of the second-order initial-value problem of (49).
Case Study 4. Consider the following second-order singular initial-value problem:

$$
\begin{gathered}
\frac{\partial^{2} u}{\partial t^{2}}+\frac{1}{t} \frac{\partial u}{\partial t}-\frac{1}{4 t^{2}} u=u \cdot \frac{\partial^{2} u}{\partial x^{2}}+4+18 t \\
u(x, 0)=0, \quad u_{t}(x, 0)=0 .
\end{gathered}
$$

The inverse differential operator $L_{t}^{-1}(\cdot)=\left(1 / t^{1 / 2}\right)$ $\int_{0}^{t} \int_{0}^{t} t^{1 / 2}(\cdot) d t d t$, developed in (31) with $p=1 / 2$, is applied on PDE of the singular initial-value problem of (55) which results in

$$
u(x, t)=\frac{16}{15} t^{2}+\frac{72}{35} t^{3}+L_{t}^{-1}\left(u \cdot u_{x x}\right)
$$

Using the MDM as in (7), (56) can be rewritten as

$$
\begin{aligned}
& \sum_{m=0}^{\infty} u_{m}(x, t) \\
& =\frac{16}{15} t^{2}+\frac{72}{35} t^{3}+L_{t}^{-1}\left(\sum_{m=0}^{\infty} A_{m}(x, t)\right) \\
& \Longrightarrow\left\{\begin{array}{l}
u_{0}(x, t)=\frac{16}{15} t^{2}, \\
u_{1}(x, t)=\frac{72}{35} t^{3}+L_{t}^{-1}\left(A_{0}(x, t)\right), \\
u_{m+1}(x, t)=L_{t}^{-1}\left(A_{m}(x, t)\right), \quad m \geq 1 .
\end{array}\right.
\end{aligned}
$$

The Adomian polynomials $A_{m}$ 's are obtained as

$$
\begin{aligned}
& A_{0}(x, t)=u_{0}(x, t) \cdot u_{0_{x x}}(x, t)=0, \\
& A_{1}(x, t)=u_{0}(x, t) \cdot u_{1_{x x}}(x, t)+u_{1}(x, t) \cdot u_{0_{x x}}(x, t)=0 \text {, } \\
& A_{2}(x, t)=u_{0}(x, t) \cdot u_{2_{x x}}(x, t)+u_{1}(x, t) \cdot u_{1_{x x}}(x, t) \\
& +u_{2}(x, t) \cdot u_{0_{x x}}(x, t)=0 \text {, } \\
& A_{m}(x, t)=0, \quad m \geq 3,
\end{aligned}
$$

and the expressions for $u_{m}$ 's become

$$
\begin{aligned}
& u_{0}(x, t)=\frac{16}{15} t^{2}, \\
& u_{1}(x, t)=\frac{72}{35} t^{3}, \\
& u_{2}(x, t)=L_{t}^{-1}\left(A_{1}(x, t)\right)=0, \\
& u_{m}(x, t)=0, \quad m \geq 3 .
\end{aligned}
$$

Thus, the solution of the second-order singular initial-value problem of (55) by the MDM using the inverse differential operator developed in (31) is as follows:

$$
u(x, t)=u_{0}(x, t)+u_{1}(x, t)+\cdots=\frac{16}{15} t^{2}+\frac{72}{35} t^{3},
$$


which is the exact solution of (55). The exact solution of (55), that is, (60), is obtained only by the first-order approximation using the new inverse differential operator developed in (31), Section 2.4. This reveals the fact that the new developed inverse differential operators are very effective tools in solving the first- and second-order singular nonlinear PDEs by the modified decomposition method.

\section{Conclusions}

The modified decomposition method (MDM) is used to solve the first- and second-order singular nonlinear partial differential equations. Four new inverse differential operators are developed and used with the first- and second-order singular nonlinear PDEs to be adapted for the modified decomposition method (MDM). A few case studies are provided for the use of the MDM for solving the singular nonlinear PDEs of initial-value problems (IVPs) which include one first-order and three second-order initial-value problems. The results of the solutions of the initial-value problems are compared with the existing exact solutions which show excellent agreement.

\section{Conflict of Interests}

The author declares that there is no conflict of interests regarding the publication of this paper.

\section{References}

[1] A.-M. Wazwaz and S. M. El-Sayed, "A new modification of the Adomian decomposition method for linear and nonlinear operators," Applied Mathematics and Computation, vol. 122, no. 3, pp. 393-405, 2001.

[2] A.-M. Wazwaz, "The modified decomposition method for analytic treatment of differential equations," Applied Mathematics and Computation, vol. 173, no. 1, pp. 165-176, 2006.

[3] M. M. Hosseini and H. Nasabzadeh, "Modified Adomian decomposition method for specific second order ordinary differential equations," Applied Mathematics and Computation, vol. 186, no. 1, pp. 117-123, 2007.

[4] L. Blanco-Cocom, A. G. Estrella, and E. Avila-Vales, "Solving delay differential systems with history functions by the Adomian decomposition method," Applied Mathematics and Computation, vol. 218, no. 10, pp. 5994-6011, 2012.

[5] A.-M. Wazwaz, "A reliable algorithm for solving boundary value problems for higher-order integro-differentiable equations," Applied Mathematics and Computation, vol.118, no. 2-3, pp. 327342, 2001.

[6] A. Ebaid, "A new analytical and numerical treatment for singular two-point boundary value problems via the Adomian decomposition method," Journal of Computational and Applied Mathematics, vol. 235, no. 8, pp. 1914-1924, 2011.

[7] W. Al-Hayani, "Adomian decomposition method with Green's function for sixth-order boundary value problems," Computers \& Mathematics with Applications, vol. 61, no. 6, pp. 1567-1575, 2011.

[8] E. H. Aly, A. Ebaid, and R. Rach, "Advances in the Adomian decomposition method for solving two-point nonlinear boundary value problems with Neumann boundary conditions,"
Computers \& Mathematics with Applications, vol. 63, no. 6, pp. 1056-1065, 2012.

[9] J.-S. Duan, T. Chaolu, and R. Rach, "Solutions of the initial value problem for nonlinear fractional ordinary differential equations by the Rach-Adomian-Meyers modified decomposition method," Applied Mathematics and Computation, vol. 218, no. 17, pp. 8370-8392, 2012.

[10] A.-M. Wazwaz and M. S. Mehanna, "The combined LaplaceAdomian method for handling singular integral equation of heat transfer," International Journal of Nonlinear Science, vol. 10, no. 2, pp. 248-252, 2010.

[11] A. Hasseine, A. Bellagoun, and H.-J. Bart, "Analytical solution of the droplet breakup equation by the Adomian decomposition method," Applied Mathematics and Computation, vol. 218, no. 5, pp. 2249-2258, 2011.

[12] M. Danish, S. Kumar, and S. Kumar, "Approximate explicit analytical expressions of friction factor for flow of Bingham fluids in smooth pipes using Adomian decomposition method," Communications in Nonlinear Science and Numerical Simulation, vol. 16, no. 1, pp. 239-251, 2011.

[13] Q. Mao, "Free vibration analysis of elastically connected multiple-beams by using the Adomian modified decomposition method," Journal of Sound and Vibration, vol. 331, no. 11, pp. 2532-2542, 2012.

[14] G. Adomian, R. Rach, and N. T. Shawagfeh, "On the analytic solution of the lane-emden equation," Foundations of Physics Letters, vol. 8, no. 2, pp. 161-181, 1995.

[15] A.-M. Wazwaz, "A new method for solving singular initial value problems in the second-order ordinary differential equations," Applied Mathematics and Computation, vol. 128, no. 1, pp. 45-57, 2002.

[16] Y. Q. Hasan and L. M. Zhu, "Modified Adomian decomposition method for singular initial value problems in the second-order ordinary differential equations," Surveys in Mathematics and its Applications, vol. 3, pp. 183-193, 2008.

[17] G.-C. Wu, "Adomian decomposition method for non-smooth initial value problems," Mathematical and Computer Modelling, vol. 54, no. 9-10, pp. 2104-2108, 2011.

[18] T. A. Abassy, "Improved Adomian decomposition method (solving nonlinear non-homogenous initial value problem)," Journal of the Franklin Institute. Engineering and Applied Mathematics, vol. 348, no. 6, pp. 1035-1051, 2011.

[19] Y. Lin, Y. Liu, and Z. Li, "Symbolic computation of analytic approximate solutions for nonlinear differential equations with initial conditions," Computer Physics Communications, vol. 183, no. 1, pp. 106-117, 2012.

[20] A.-M. Wazwaz, R. Rach, and J.-S. Duan, "Adomian decomposition method for solving the Volterra integral form of the LaneEmden equations with initial values and boundary conditions," Applied Mathematics and Computation, vol. 219, no. 10, pp. 5004-5019, 2013. 


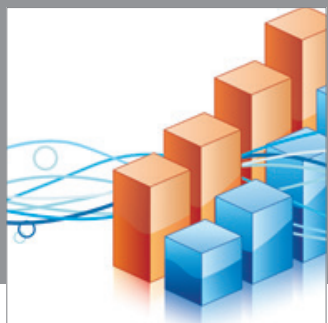

Advances in

Operations Research

mansans

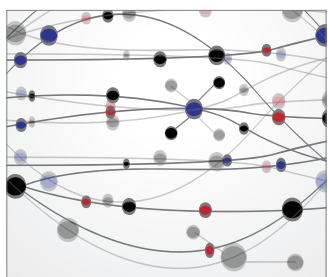

The Scientific World Journal
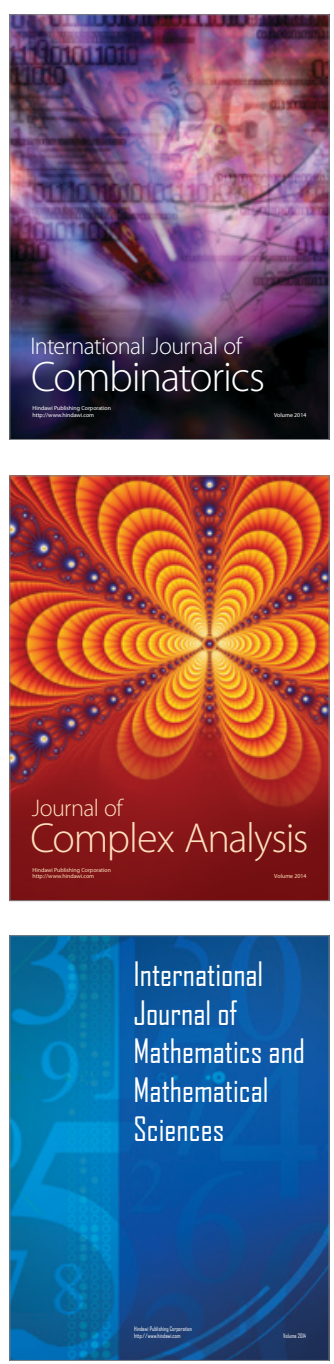
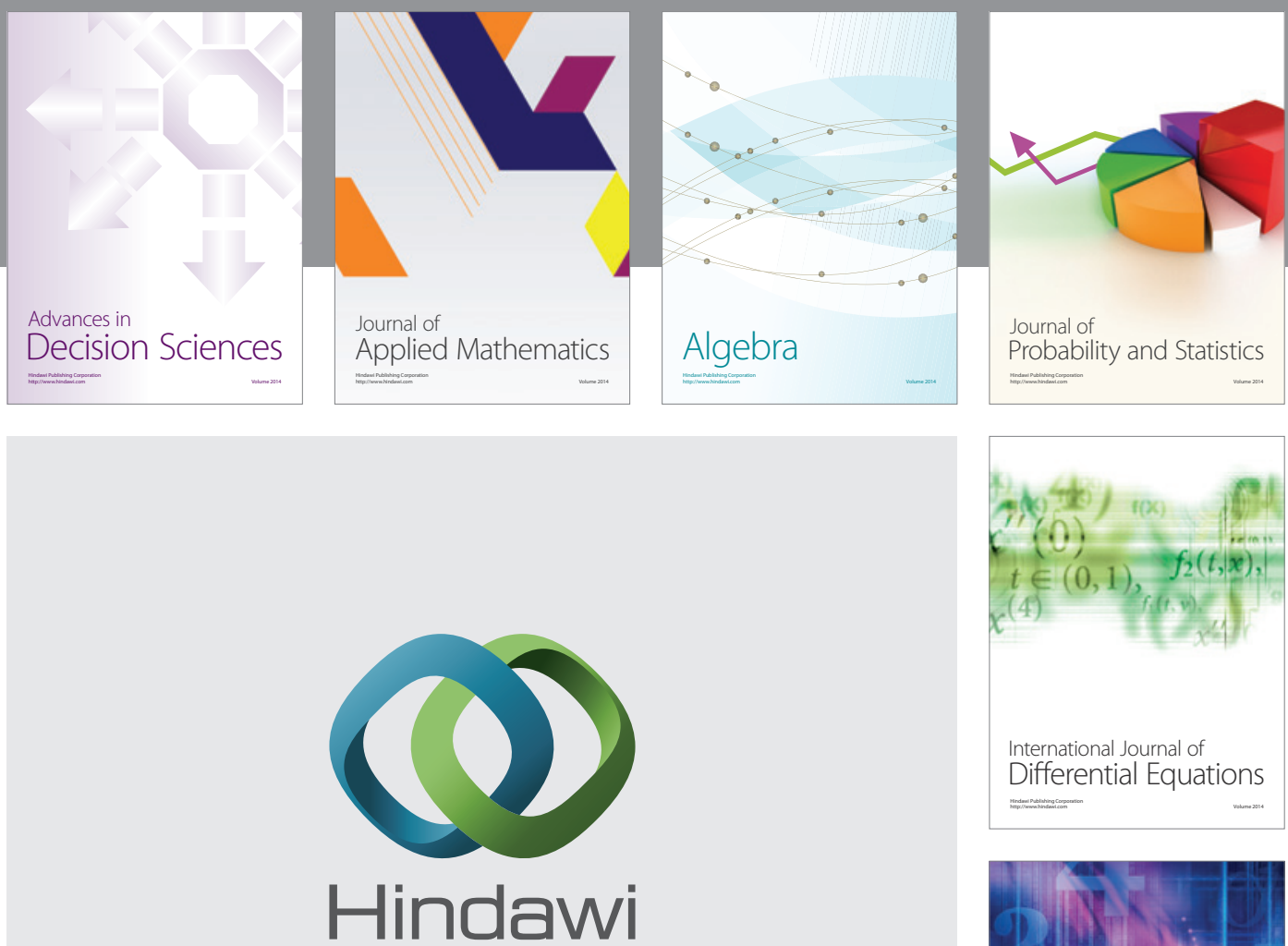

Submit your manuscripts at http://www.hindawi.com
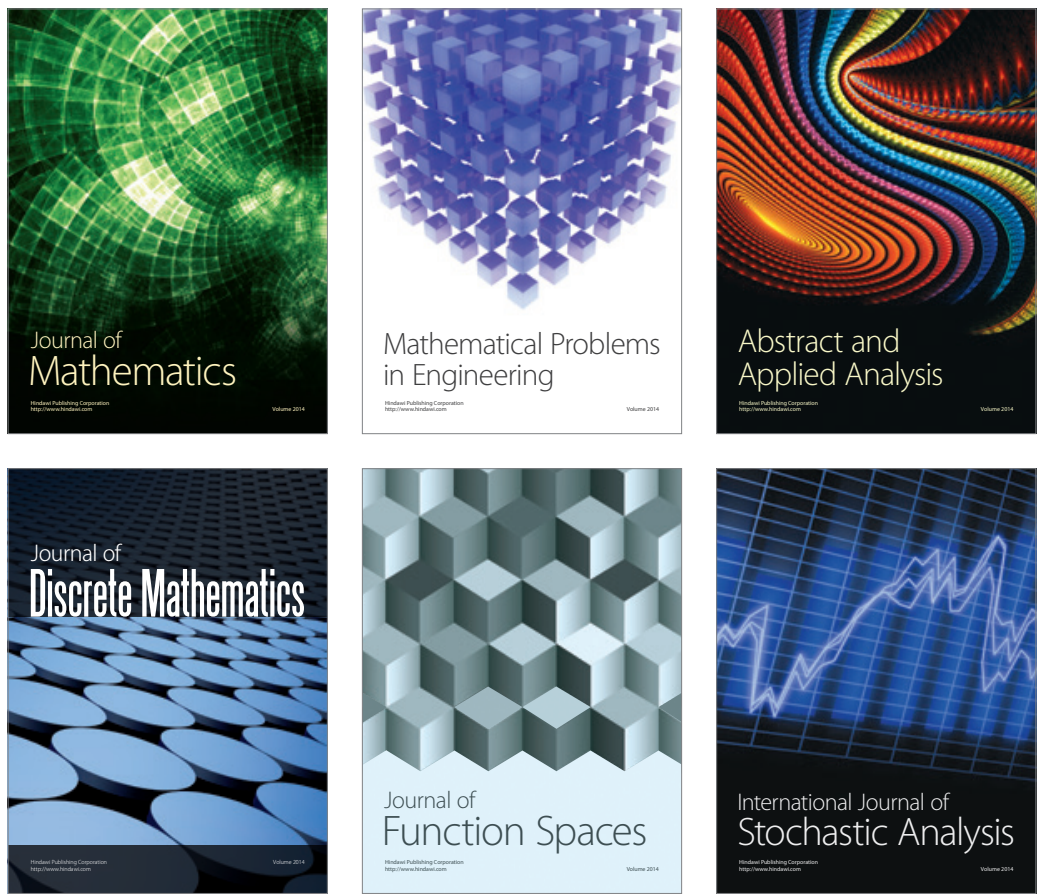

Journal of

Function Spaces

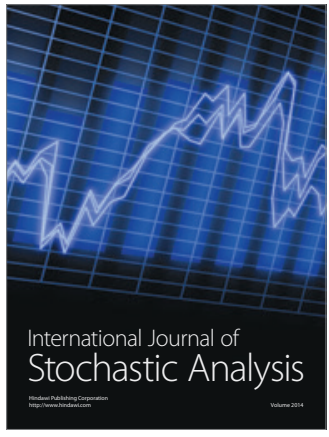

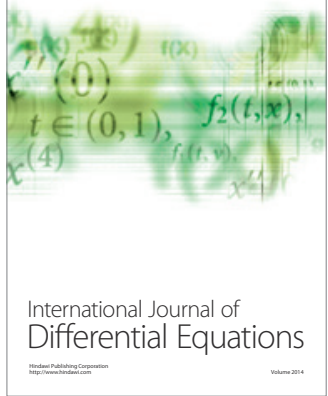
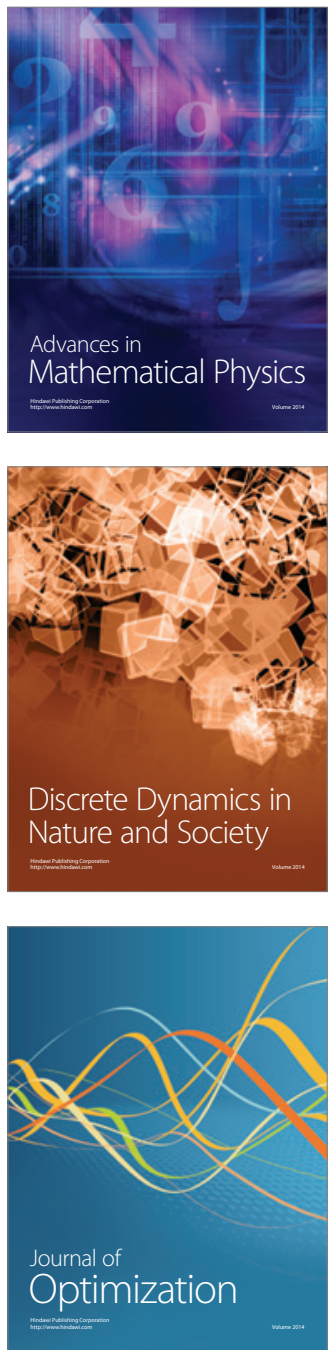